\title{
Desempenho de um trator em função do tipo de pneu, da lastragem e da velocidade de trabalho
}

\author{
Performance of an tractor as a function of tire type, ballasting and forward speed
}

\author{
Afonso Lopes ${ }^{1}$ Kléber Pereira Lanças ${ }^{2}$ Rouverson Pereira da Silva ${ }^{1}$ \\ Carlos Eduardo Angeli Furlani ${ }^{1}$ Alberto Kazushi Nagaoka ${ }^{3}$ \\ Gustavo Naves dos Reis ${ }^{4}$
}

\section{RESUMO}

O trabalho comparou o desempenho de um trator agrícola 4x2 TDA de $89 \mathrm{~kW}$ (121 cv) em função do tipo de pneus (radial, diagonal e de baixa pressão), a condição de lastragem (com e sem água nos pneus) e quatro velocidades [V1 $\left(1,84 \mathrm{~km} \mathrm{~h}^{-1}\right)$, $V 2\left(3,18 \mathrm{~km} \mathrm{~h}^{-1}, V 3\left(4,57 \mathrm{~km} \mathrm{~h}^{-1}\right), V 4\left(5,04 \mathrm{~km} \mathrm{~h}^{-1}\right)\right]$. O experimento foi realizado na UNESP-Jaboticabal-SP, em condição de preparo do solo com escarificador de sete hastes a $30 \mathrm{~cm}$ de profundidade. Os pneus foram do tipo R1, com as seguintes características: [radial (dianteiros-14.9 $R$ 26; traseiros-620/75 $R$ 30) diagonal (dianteiros-14.9-26, traseiros-23.1-30) e BPAF (dianteiros-500/ 60-26.5; traseiros-700/55-34)]. $O$ delineamento experimental foi blocos casualizados, em esquema fatorial $3 \times 2 \times 4$, com 24 tratamentos e três repetições. Os resultados evidenciaram vantagens para o trator equipado com pneus radiais.

Palavras-chave: escarificação, lastro, preparo do solo.

\section{ABSTRACT}

The objective of this study was to compare the performance of the FWD agricultural tractor, equipped with three types of tires (bias ply, radial and low pressure), with and without tire water ballasting, operating in four forward speed. The research was carried out at UNESP, SP, Brazil, with the tractor pulling a chisel plow. A tractor with $89 \mathrm{~kW}(121 \mathrm{cv})$ engine power equipped with FWD, was used in the experiment, and the tires used had the following characteristics: radial (front: $14.9 R 26$ and rear: 620/75R30), bias ply (front: 14.9-26 and rear: 23.130) and low pressure (front: 500/60-26.5 and rear: 700/55-34). The experiment was conducted in a completely randomized block, design (3x2x4), with 24 treatments and three replications. The results evidenced advantages for the tractor equipped with radial tires.

Key words: chiseling, ballating, soil tillage.

\section{INTRODUÇÃO}

Várias indagações ocorrem sobre o efeito positivo ou negativo no desempenho de tração, quando se varia o tipo construtivo dos rodados pneumáticos dos tratores. CORRRÊA (1993) comparou o desempenho de tração entre rodados de construção radial e diagonal, com patinagem entre 0 a 30\%, em duas condições de superfícies, encontrando melhores características de tração (força na barra, potência na barra e coeficiente de tração) para os pneus de construção radial. GEE-CLOUGH et al. (1977), estudando pneus de construção radial e diagonal em condições de campo, num período de aproximadamente três anos, concluíram que a construção radial resulta em um aumento de 5 a $8 \%$ na razão dinâmica de tração para uma patinagem média de até $20 \%$.

Em estudo do comportamento de tração com rodados pneumáticos de construção diagonal e radial em condições de solo arenoso, argiloso, argilo-

'Professor do Departamento de Engenharia Rural, Universidade Estadual Paulista (UNESP), Campus de Jaboticabal, 14884-900, Jaboticabal, SP. Fone: 16-3209-2637. E-mail: afonso@fcav.unesp.br.

${ }^{2}$ Professor do Departamento de Engenharia Rural, UNESP, Campus de Botucatu, SP.

${ }^{3}$ Professor do Departamento de Engenharia Rural, Universidade do Estado de Santa Catarina, Lages, SC.

${ }^{4}$ Pós-graduando, UNESP, Campus de Jaboticabal, SP. 
arenoso e concreto, FORREST et al. (1962) concluíram que o uso de pneu radial resultou em 8 , 23, 21 e $33 \%$ a mais de força de tração quando comparado com o pneu diagonal. Segundo UPADHYAYA \& WULFSOHN (1989), o tipo construtivo dos pneus influencia a durabilidade e o desempenho de tração dos tratores. Os autores compararam pneus radiais e diagonais em vários tipos de solo e chegaram à conclusão de que, em relação à tração, as condições do solo são mais importantes que o tipo de pneu.

Procurando verificar o desempenho de um trator agrícola $4 \times 2$ TDA em condições de campo, quando são alteradas as dimensões de seu rodado de tração, MOLINA JÚNIOR et al. (1997) analisaram a força de tração desenvolvida, patinagem, potência na barra, velocidade de deslocamento, coeficiente de tração e capacidade de campo para três jogos de pneus diagonais de medidas 18.4-30, 18.4-34 e 13.6-38. Os resultados obtidos mostraram que a alteração das dimensões dos pneus interferiu no desempenho do trator, pois quando o mesmo foi equipado com o conjunto de pneus 18.4-30, apresentou menor desempenho para potência na barra, patinagem, velocidade de deslocamento, coeficiente de tração e capacidade de campo. Todavia, o conjunto de pneus 13.6-38 proporcionou a maior eficiência com o maior coeficiente de tração. Entretanto, os autores ressaltam que esses resultados não são definitivos, sendo necessária a realização de estudos em outras condições de solo.

Ao comparar a resistência ao rolamento de um trator agrícola com tração dianteira auxiliar operando com pneus radiais, diagonais e de baixa pressão e alta flutuação (BPAF), LANÇAS et al. (2000) encontraram vantagens para o pneu radial.

Evidências significativas da carga no rodado sobre os parâmetros patinagem e coeficiente de tração foram observadas por YANAI (1994), que utilizou um trator com tração dianteira auxiliar, pneus diagonais em testes realizados em condição de campo com solo não mobilizado, aplicando quatro quantidades distintas de carga sobre o rodado, que foi inflado com quatro níveis de pressão. Os resultados mostraram que a pressão de inflação teve influência significativa na velocidade de deslocamento, patinagem e potência na barra de tração, sem, no entanto, apresentar uma tendência de comportamento. A interação entre pressão de inflação e carga sobre o rodado mostrou que determinadas combinações desses dois fatores foram mais favoráveis para o desenvolvimento de maior velocidade de deslocamento e menor patinagem.
Objetivou-se avaliar o desempenho de um trator agrícola 4x2 TDA de $89 \mathrm{~kW}(121 \mathrm{cv})$, variando o tipo de pneu (radial, diagonal e baixa pressão), combinando duas condições de lastragem do trator (com e sem água nos pneus) e quatro velocidades de deslocamento.

\section{MATERIAL E MÉTODOS}

O experimento foi conduzido na UNESP, Campus de Jaboticabal, SP, cuja localização geográfica está definida pelas coordenadas $21^{\circ} 15^{\prime} 22^{\prime}$ " latitude Sul e 48 18 '58" longitude Oeste, sendo a altitude média $570 \mathrm{~m}$, e declividade média do terreno de aproximadamente $7 \%$.

A área experimental estava ocupada pela cultura de soja, sendo a mesma colhida mecanicamente, e a matéria seca $\left(2172 \mathrm{~kg} \mathrm{ha}^{-1}\right)$ distribuída na área pela ação da colhedora. Para determinação da matéria seca, utilizou-se um quadrado de $1 \mathrm{~m}$ de lado. O solo foi classificado como Latossolo Vermelho eutroférrico, A moderado, caulinítico-oxálico e relevo suave ondulado (ANDRIOLI \& CENTURION, 1999). No momento dos testes, o teor médio de água no solo foi de 22,8\%, sendo determinado de acordo com o método gravimétrico (EMBRAPA, 1997).

Os pneus de teste foram montados em um trator agrícola, 4x2TDA, de $89 \mathrm{~kW}(121 \mathrm{cv})$ de potência máxima no motor. O escarificador utilizado foi do tipo de arrasto, conjugado com rolo destorroador e discos de corte, com sete hastes retas inclinadas e ponteiras sem asa com $7 \mathrm{~cm}$ de largura. A escarificação ocorreu a $30 \mathrm{~cm}$ de profundidade média, com espaço entre hastes de $39 \mathrm{~cm}$. As marchas selecionadas para o ensaio foram M1, M2, M3 e M4, correspondendo às velocidades de $1,84 \mathrm{~km} \mathrm{~h}^{-1}, 3,18 \mathrm{~km} \mathrm{~h}^{-1}, 4,57 \mathrm{~km} \mathrm{~h}^{-1}$ e $5,04 \mathrm{~km} \mathrm{~h}^{-1}$, respectivamente.

O trator equipado com os pneus de teste, com toda a instrumentação necessária, operou com rotação de 2150rpm no motor, o que proporcionou 540rpm na tomada de potência, quando o mesmo funcionava sem carga na barra de tração. Com a finalidade de iniciar os testes com a rotação mais uniforme possível, foi desenvolvido um dispositivo que funcionou como batente quando acoplado junto com a alavanca do acelerador. Desta forma, no início de cada parcela, a alavanca de aceleração foi posicionada no batente; em seguida acionava-se o cronômetro por 20 segundos e determinava-se a rotação da TDP. Dependendo do resultado, esse dispositivo permitia um ajuste na aceleração de modo que a rotação da TDP se aproximasse de 540rpm. 
Foram utilizados três conjuntos de pneus de tração do tipo R1, os quais são: quatro radiais, quatro diagonais e quatro BPAF (baixa pressão e alta flutuação). A montagem e uso dos pneus seguiram a orientação da ASSOCIAÇÃO BRASILEIRA DE PNEUS E AROS (1994): radial (dianteiros: $14.9 \mathrm{R}$ 26; traseiros: 620/75 R 30) diagonal (dianteiros: 14.926, traseiros: 23.1-30) e BPAF (dianteiros: 500/6026.5; traseiros: 700/55-34). A força de tração na barra foi obtida por meio de célula de carga de $100 \mathrm{kN}$, e toda a instrumentação utilizada nesse trabalho encontra-se descrita conforme SILVA \& BENEZ (1997).

O trabalho foi conduzido com o trator operando em duas condições de lastragem (pneus sem água e pneus com água), ocupando $75 \%$ do volume. A massa total do trator, em condição estática pode ser vista na tabela 1 .

$\mathrm{O}$ experimento foi conduzido em blocos casualizados, com esquema fatorial $3 \times 4 \times 2$, totalizando 24 tratamentos e com três repetições. Os tratamentos foram formados pelas combinações de três tipos de pneus, quatro marchas do trator e duas condições de lastragem (com e sem água nos pneus). Cada parcela teve $30 \mathrm{~m}$ de comprimento e, entre as parcelas, na direção longitudinal, deixou-se um intervalo de $15 \mathrm{~m}$ para realizar manobras, trânsito de máquinas e estabilizar as determinações. Para comparar os tratamentos, avaliou-se a potência na barra, capacidade de campo efetiva e rotação do motor. Os dados obtidos foram submetidos à análise de variância, pelo teste $\mathrm{F}$, e a comparação de médias, pelo teste de Tukey, a 5\% de probabilidade de erro.

A potência média na barra de tração foi determinada de forma indireta; para tanto, utilizou-se a seguinte equação:

em que,

$$
P B=\frac{F T^{*} v}{3600}
$$

$\mathrm{PB}=$ potência na barra de tração $(\mathrm{kW})$;

$\mathrm{FT}$ = força média de tração na barra $(\mathrm{N})$; e

$\mathrm{v}=$ velocidade real de deslocamento $\left(\mathrm{km} \mathrm{h}^{-1}\right)$.

A capacidade de campo efetiva foi calculada a partir da largura de corte média real do escarificador $(2,73 \mathrm{~m})$ e da velocidade real de deslocamento do conjunto. A largura de corte foi considerada fixa de $2,73 \mathrm{~m}$, uma vez que se manteve o mesmo número de hastes, mesma profundidade de trabalho e mesma relação espaçamento entre hastes/profundidade de trabalho.

Para o cálculo da capacidade de campo efetiva utilizou-se a seguinte equação: em que,

$$
C C E=\frac{v^{*} L m}{10}
$$

CCE = capacidade de campo efetiva $\left(\right.$ ha $\left.h^{-1}\right)$;

$\mathrm{v}=$ velocidade real de deslocamento $\left(\mathrm{km} \mathrm{h}^{-1}\right)$;

$\mathrm{Lm}=$ largura real de corte do implemento (m), e

$10=$ fator de conversão.

A rotação do motor foi determinada de forma indireta, utilizando-se a rotação da tomada de potência e a relação de transmissão do trator. Utilizouse a seguinte equação:

\section{$R M=R T D P * R T$}

em que,

$\mathrm{RM}=$ rotação do motor (rpm),

RTDP $=$ rotação da TDP $(\mathrm{rpm})$, e

$\mathrm{RT}$ = relação de transmissão do trator de teste, entre o motor e a TDP $(3,9815)$.

\section{RESULTADOS E DISCUSSÃO}

Observa-se que, para a potência na barra, ocorreram interações entre os fatores, para as interações entre pneu e marcha e entre lastro e marcha (Tabela 2). O desdobramento destas interações indicou que, para as marchas M1 e M2, não houve diferença entre todos os pneus estudados (Tabela 3), enquanto que para as marchas M3 e M4, o pneu radial teve desempenho superior aos demais. Esses resultados estão de acordo com CORRÊA (1993), que também evidenciou vantagens para o pneu radial.

A análise da tabela 3 revela ainda que, para o pneu radial e de baixa pressão, a variável potência na barra foi diferente estatisticamente para todas as marchas ensaiadas, ao passo que para o pneu diagonal, observou-se diferença nas marchas M1, M2 e M3, sendo essa última semelhante à marcha M4.

Com relação ao desdobramento da interação entre lastro e marcha, mostrada na tabela 4, nota-se que, para as marchas M1 e M4, ocorreu semelhança entre as condições de lastragem (sem água e com água nos pneus) para variável potência na barra. Nas marchas M2 e M3, a condição de lastragem com água foi estatisticamente superior. Observa-se também que para as duas condições de lastragem, a variável potência na barra teve diferença significativa em todas as marchas ensaiadas.

Tabela 1 - Massa total do trator $(\mathrm{kg})$ em condição estática.

\begin{tabular}{lccc}
\hline Lastro & Pneu radial & Pneu diagonal & Pneu BPAF \\
\hline Sem água & 5310 & 5382 & 5624 \\
Com água & 6788 & 6830 & 7060 \\
\hline
\end{tabular}

Ciência Rural, v.35, n.2, mar-abr, 2005. 
Tabela 2 - Síntese da análise de variância e do teste de médias ${ }^{\#}$ para as variáveis de potência na barra, capacidade de campo efetiva e rotação do motor.

\begin{tabular}{|c|c|c|c|}
\hline Fatores & $\begin{array}{c}\text { Potência na } \\
\text { barra }(\mathrm{kW})\end{array}$ & $\begin{array}{c}\text { Capacidade de campo } \\
\text { efetiva }\left(\mathrm{ha} \mathrm{h}^{-1}\right)\end{array}$ & $\begin{array}{l}\text { Rotação do } \\
\text { motor (rpm) }\end{array}$ \\
\hline \multicolumn{4}{|l|}{ Pneu (P) } \\
\hline Radial & 30,09 & $1,04 \mathrm{~b}$ & $1966 \mathrm{~b}$ \\
\hline Diagonal & 25,95 & $0,97 \mathrm{a}$ & $1867 \mathrm{a}$ \\
\hline Baixa pressão & 26,72 & $0,97 \mathrm{a}$ & $2015 \mathrm{~b}$ \\
\hline \multicolumn{4}{|l|}{ Lastro (L) } \\
\hline Pneu sem água & 24,99 & 0,95 & 1960 a \\
\hline Pneu com água & 30,17 & 1,04 & $1937 \mathrm{a}$ \\
\hline \multicolumn{4}{|l|}{$\operatorname{Marcha}(\mathrm{M})$} \\
\hline M1 & 12,19 & 0,50 & $2119 d$ \\
\hline M2 & 23,07 & 0,87 & 2026 c \\
\hline M3 & 35,18 & 1,24 & $1935 \mathrm{~b}$ \\
\hline M4 & 39,91 & 1,37 & $1713 \mathrm{a}$ \\
\hline \multicolumn{4}{|l|}{ Teste F } \\
\hline $\mathrm{P}$ & $12,63 *$ & $9,46 *$ & $14,67 *$ \\
\hline $\mathrm{L}$ & $52,48 *$ & $30,81 *$ & $1,02 \mathrm{NS}$ \\
\hline M & $304,83 *$ & $652,37 *$ & $56,55 *$ \\
\hline PxL & $0,42 \mathrm{NS}$ & $1,79 \mathrm{NS}$ & $2,71 \mathrm{NS}$ \\
\hline PxM & $2,33 *$ & $1,98 \mathrm{NS}$ & $0,81 \mathrm{NS}$ \\
\hline LxM & $3,11 *$ & $2,94 *$ & $2,19 \mathrm{NS}$ \\
\hline PxLxM & $0,35 \mathrm{NS}$ & $3,06 *$ & $0,95 \mathrm{NS}$ \\
\hline $\mathrm{CV} \%$ & 10,99 & 6,54 & 5,04 \\
\hline
\end{tabular}

\# Em cada coluna, para cada fator, médias não seguidas de mesma letra minúscula diferem pelo teste de Tukey, a $5 \%$ de probabilidade de erro, e média desacompanhada de letra implica interação entre os fatores

- NS: Não significativo.

- *: Significativo a $5 \%$ de probabilidade de erro.

- CV: Coeficiente de variação.

A semelhança de comportamento observada em M1 e M4 é devido ao alto e baixo torque no rodado respectivamente, sendo estas velocidades não recomendadas do ponto de vista operacional. As diferenças observadas em M2 e M3 ocorreram devido a tais marchas serem aquelas recomendadas para este tipo de operação.

Tabela 3 - Dados da interação (pneu e marcha) para a potência na barra de tração $(\mathrm{kW})$.

\begin{tabular}{lcccc}
\hline \multirow{4}{*}{ Pneu } & \multicolumn{4}{c}{ Marchas Ensaiadas } \\
\cline { 2 - 5 } & $\begin{array}{c}\text { M1 } \\
\left(1,84 \mathrm{~km} \mathrm{~h}^{-1}\right)\end{array}$ & $\begin{array}{c}\text { M2 } \\
\left(3,18 \mathrm{~km} \mathrm{~h}^{-1}\right)\end{array}$ & $\begin{array}{c}\text { M3 } \\
\left(4,57 \mathrm{~km} \mathrm{~h}^{-1}\right)\end{array}$ & $\begin{array}{c}\text { M4 } \\
\left(5,04 \mathrm{~km} \mathrm{~h}^{-1}\right)\end{array}$ \\
\hline Radial & $13,10 \mathrm{a} \mathrm{A}$ & 23,87 a B & $38,83 \mathrm{~b} \mathrm{C}$ & $44,55 \mathrm{~b} \mathrm{D}$ \\
Diagonal & 11,72 a A & 22,66 a B & 35,51 a C & 35,93 a C \\
BPAF & 11,77 a A & 22,67 a B & 33,20 a C & 39,24 a D \\
\hline
\end{tabular}

- Médias não seguidas por mesma letra, minúscula na coluna e maiúscula na linha, diferem pelo teste de Tukey a $5 \%$ de probabilidade de erro.
Tabela 4 - Interação 2 (lastro e marcha) para a potência na barra de tração $(\mathrm{kW})$.

\begin{tabular}{|c|c|c|c|c|}
\hline \multirow{3}{*}{ Lastro } & \multicolumn{4}{|c|}{ Marchas ensaiadas } \\
\hline & M1 & M2 & M3 & M4 \\
\hline & $\left(1,84 \mathrm{~km} \mathrm{~h}^{-1}\right)$ & $\left(3,18 \mathrm{~km} \mathrm{~h}^{-1}\right)$ & $\left(4,57 \mathrm{~km} \mathrm{~h}^{-1}\right)$ & $\left(5,04 \mathrm{~km} \mathrm{~h}^{-1}\right)$ \\
\hline Sem água & 11,06 a A & 21,06 a B & 31,94 a C & 35,94 a D \\
\hline Com água & 13,33 a $A$ & $25,07 \mathrm{~b}$ B & $38,41 \mathrm{~b} \mathrm{C}$ & 43,88 a D \\
\hline
\end{tabular}

- Médias não seguidas por mesma letra, minúscula na coluna e maiúscula na linha, diferem pelo teste de Tukey a $5 \%$ de probabilidade de erro.

Observando-se os resultados de capacidade de campo efetiva (Tabela 2), percebe-se que o fator marcha foi influenciado pelo fator lastro, ou ocorreu interação estatística significativa. Já o fator pneu influenciou significativamente a variável capacidade de campo efetiva, observando-se o maior valor para o pneu radial, enquanto o pneu diagonal foi semelhante ao de baixa pressão.

Nos dados da interação entre os fatores lastro e marcha, cada valor representa média de nove observações, mostrando que, para as marchas M1 e M2, ocorreu semelhança entre as condições de lastragem sem água e com água nos pneus para a variável capacidade de campo efetiva; para M3 e M4, a condição de lastragem com água foi superior (Tabela 5). Essa diferença se explica devido ao fato de, nas marchas M3 e M4, o fator lastro resultar em mais acréscimo de velocidade. Observa-se ainda que, para as duas condições de lastragem, a variável capacidade de campo efetiva teve diferença significativa para todas as marchas testadas e o aumento ocorreu de M1 para M4.

Para a rotação do motor, de acordo com os dados da tabela 2, percebe-se que não ocorreu interação entre os fatores. Pela síntese do teste F, verifica-se que o tipo de pneu influenciou a rotação do motor, sendo o pneu radial semelhante ao pneu de baixa pressão e os dois apresentaram resultados

Tabela 5 - Interação entre os fatores lastro e marcha para a variável capacidade de campo efetiva $\left(\mathrm{ha} \mathrm{h}^{-1}\right)$.

\begin{tabular}{lcccc}
\hline \multirow{4}{*}{ Lastro } & \multicolumn{4}{c}{ Marchas ensaiadas } \\
\cline { 2 - 5 } & $\begin{array}{c}\mathrm{M} 1 \\
\left(1,84 \mathrm{~km} \mathrm{~h}^{-1}\right)\end{array}$ & $\begin{array}{c}\text { M2 } \\
\left(3,18 \mathrm{~km} \mathrm{~h}^{-1}\right)\end{array}$ & $\begin{array}{c}\text { M3 } \\
\left(4,57 \mathrm{~km} \mathrm{~h}^{-1}\right)\end{array}$ & $\begin{array}{c}\text { M4 } \\
\left(5,04 \mathrm{~km} \mathrm{~h}^{-1}\right)\end{array}$ \\
\hline Sem água & $0,48 \mathrm{a} \mathrm{A}^{2}$ & $0,84 \mathrm{a} \mathrm{B}$ & $1,21 \mathrm{a} \mathrm{C}$ & $1,29 \mathrm{a} \mathrm{D}$ \\
Com água & $0,53 \mathrm{a} \mathrm{A}$ & $0,89 \mathrm{a} \mathrm{B}$ & $1,29 \mathrm{~b} \mathrm{C}$ & $1,45 \mathrm{~b} \mathrm{D}$ \\
\hline
\end{tabular}

- Médias não seguidas por mesma letra, minúscula na coluna e maiúscula na linha, diferem pelo teste de Tukey a $5 \%$ de probabilidade de erro. 
superiores ao pneu diagonal. Observa-se também que o fator lastro não resultou em diferença para a variável rotação do motor. $\mathrm{O}$ fator marcha influenciou a variável rotação do motor causando diferença para todas as marchas ensaiadas independentemente do tipo de pneu.

\section{CONCLUSÕES}

A combinação do tipo de pneu e marcha selecionada evidenciou a superioridade do pneu radial para a variável potência na barra, operando com o trator na marcha M3 $\left(4,57 \mathrm{~km} \mathrm{~h}^{-1}\right)$.

A combinação da condição de lastragem e a marcha selecionada para as variáveis potências na barra e capacidade de campo efetiva reforçam a importância da lastragem com líquido nos pneus do trator para trabalhar a $4,57 \mathrm{~km} \mathrm{~h}^{-1}$ (M3) em uma operação de preparo do solo com escarificador.

\section{REFERÊNCIAS BIBLIOGRÁFICAS}

ASSOCIAÇÃO BRASILEIRA DE PNEUS E AROS. Livro de normas técnicas. São Paulo, 1994. 108p.

ANDRIOLI, I.; CENTURION, J.F. Levantamento detalhado dos solos da Faculdade de Ciências Agrárias e Veterinárias de Jaboticabal. In: CONGRESSO BRASILEIRO DE CIÊNCIA DO SOLO, 27., 1999, Brasília. Anais... Brasília : Sociedade Brasileira de Ciência do Solo, 1999. 32p. (T025-3 CD-ROM)

CORRÊA, I.M. Características de tração de um trator agrícola utilizando-se de pneus radial e diagonal. 1993. 99f. Dissertação (Mestrado em Agronomia) - Curso de Pós-graduação em Máquinas Agrícolas, Escola Superior de Agricultura "Luiz de Queiroz”, Universidade de São Paulo.

EMBRAPA - EMPRESA BRASILEIRA DE PESQUISA AGROPECUÁRIA. Centro Nacional de Pesquisa de Solos. Manual de métodos de análise do solo. Rio de Janeiro, 1997. 212p.

FORREST, P.J. et al. Tractive characteristics of radial-ply tires. Transactions of ASAE, St. Joseph, v.5, n.2, p.108-115, 1962.

GEE-CLOUGH, D. et al. Tractive performance of drive tyres. II A comparison of radial and cross-ply carcass construction. Journal of Agriculture Engineering Research, v.22, p.385-395, 1977.

LANÇAS, K.P. et al. Avaliação da resistência ao rolamento do rodado de trator agrícola em função do tipo construtivo, da lastragem e da velocidade de deslocamento em condição de campo. Engenharia Agrícola, Jaboticabal, v.20, n.2, p.171-178, 2000.

MOLINA JÚNIOR, W.F. et al. Capacidade de tração dos tratores agrícolas devido à variação de medida dos pneus. In: CONGRESSO BRASILEIRO DE ENGENHARIA AGRÍCOLA, 26., 1997, Campina Grande. CD ROM... Campina Grande : SBEA, 1997. MAG065.

SILVA, S.L.; BENEZ, S.H. Construção de sistema de aquisição de dados para avaliação do desempenho energético de máquinas e implementos agrícolas em ensaios de campo. 1997. Energia na Agricultura, Botucatu, v.12, n.3, p.10-18, 1997.

UPADHYAYA, S.K.; WULFSOHN, D. An overview of traction research at university of California, Davis. Califórnia Agriculture, v.43, n.2, 1989.

YANAI, K. Influência da pressão de inflação e da carga sobre o rodado no desempenho operacional de trator com e sem o acionamento da tração dianteira auxiliar. 1994. 69f. Dissertação (Mestrado em Agronomia) - Pós-graduação em Energia na Agricultura, Faculdade de Ciências Agrárias, Universidade Estadual Paulista. 